\title{
An Approach to Integrate Heterogeneous Web Applications
}

\author{
Saurabh Rawat
}

Anushree Sah

\begin{abstract}
As the market is changing with high speed so the need is also changing. As the technologies like Http, SOAP, UDDI etc came into existence and also www (World Wide Web) gained publicity, so the need for applications over internet emerges. And SOA and web services provide a solution to this problem. They help us in making interoperable software systems for both small businesses or for huge enterprise applications. This design framework (SOA) will also increase reusability, granularity, interoperability and modularity of software applications over internet.
\end{abstract}

This paper discuses the integration of different applications that will use these technologies in real world. It will explore different ways to interact between heterogeneous applications made in different languages like ASP.NET, Java, Silverlight and also with the database MS SQL Server 2000. We will also discuss about XML (EXTENSIBLE MARKUP LANGUAGE) which is Language for communication between different web services and XSLT (Extensible Style sheet Language Transformations) in next paper.

In this paper, we describe the formatting guidelines for IJCA Journal Submission.

\section{Keywords}

Keywords: SOA, ASP.NET, JAVA, XML, XSLT, Software as a Service, Application integration and WEB SERVICES.

\section{INTRODUCTION}

Now a day's software industry is involved in the development of new internet enabled systems, the integration of legacy systems into intranets or extranets, and advanced internet publishing. There is high need for designing, developing and maintaining such systems in most effective and efficient way. Almost all the businesses are using web or are in process of adapting web. B2B (Business to Business) interactions are dominant in today's world and the usage of B2B is increasing the application to application interactions so the need for using web services is also increasing in today's emerging market. Our work is a part of a larger project that provides a common platform for B2B interactions and thus building internet enabled enterprise systems. We have taken a case study into consideration to show how in real world different applications are communicating with each other. This case study is an approach to use web services in real world and is more widely used and accepted.

\section{EVALUATION AND ANALYSIS OF LITERATURE READ}

For doing this case study we have to read and understand following topics. There is an abstract of all the terminologies or technologies we will be using. After understanding these topics it will be easier to understand my work.

\section{SOA: SERVICE ORIENTED ARCHITECHTURE}

Systems are getting bigger and bigger than ever before. So complexity is also increasing with them. As said by Juval Lowey "Complexity increases exponentially with size". And systems need to be interconnected with each other. So for interconnecting these systems many concepts evolved and more will come as per the demands in future. "Like OO (Object Oriented) solved the problems of small-medium sized systems. CO (Component Orientation) solved problems OO couldn't on medium-large systems. Neither OO nor CO could cope with the problems of very large systems, systems of systems, or integration between systems. So SOA evolved. SOA attempts to solve problems $\mathrm{OO}$ and $\mathrm{CO}$ could not solve by raising the level of abstraction. It is an architectural style and a design principle for application development and integration. Or we can say it is a way of designing software systems to provide "services" to end-user applications or to other services. SOA is a natural evolutionary step to the object-oriented (OO), procedural, and data-centric approaches adopted for solution implementation till now. [4]

\section{WEB SERCIVES:}

The W3C defines a "Web service" as "a software system designed to support interoperable machine-to-machine interaction over a network. It has an interface described in a machine-processable format (specifically Web Services Description Language WSDL). Other systems interact with the Web service in a manner prescribed by its description using SOAP messages, typically conveyed using HTTP with an XML serialization in conjunction with other Web-related standards."

The W3C also states, "We can identify two major classes of Web services, REST-compliant Web services, in which the primary purpose of the service is to manipulate XML representations of Web resources using a uniform set of "stateless" operations; and arbitrary Web services, in which the service may expose an arbitrary set of operations."[2]. We will use both of these web services in our application and try to integrate our heterogeneous web applications using them. 

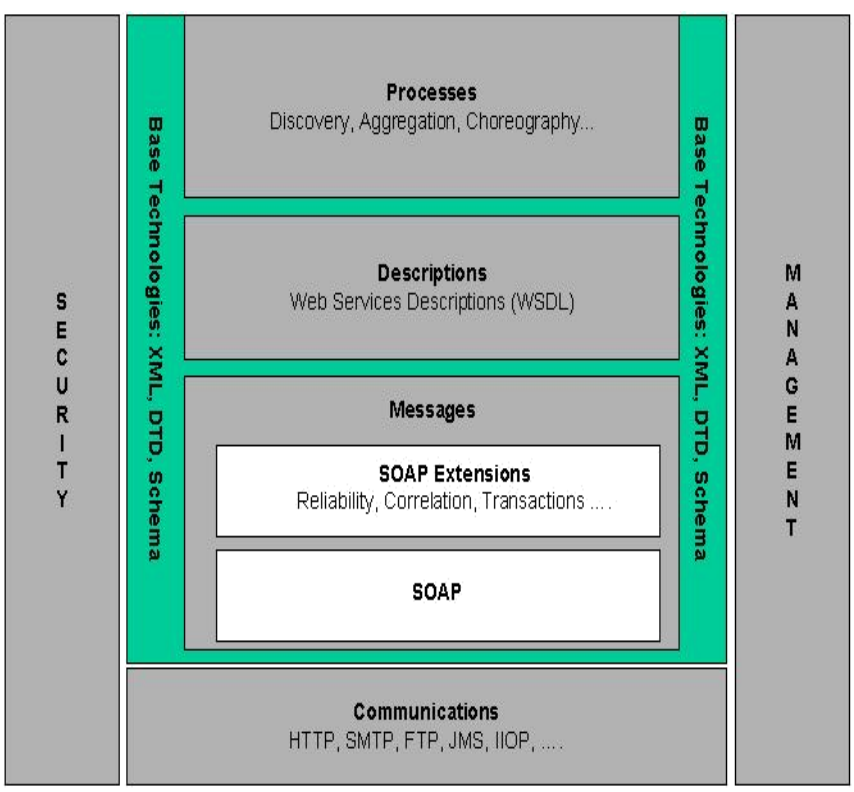

Fig 1. Web Services Architecture Stack[2]

\section{WSDL (Web Services Description Language)}

WSDL stands for Web Services Description Language. It is written in XML and is a XML document. WSDL is used to describe Web services. It is a contract between the Service Requestor and Service Provider. WSDL is also used to locate Web services over World Wide Web. Operations and messages performed/understood are described abstractly in it. You may also say it is endpoints for accessing the services. It gives Transport Binding Information - how messages are transported over internet. WSDL describes the SOAP Messages.[5]

\section{Simple Object Access Protocol}

SOAP (Simple Object Access Protocol) is a lightweight XML-based protocol used to exchange information in a decentralized, distributed environment. It is an XML based standard for distributed messaging and the message format is independent of the transport protocol. SOAP messages can be delivered via a variety of transports including: $\operatorname{HTTP}(\mathrm{S})$, SMTP, Message Oriented Middleware etc.

\section{Microsoft SQL Server}

Microsoft SQL Server is a relational database management system (RDBMS) produced by Microsoft. Its primary query language is Transact-SQL, an implementation of the ANSI/ISO standard Structured Query Language (SQL) used by both Microsoft and Sybase[6].

\section{APPROACH}

In this section we present an overview of our approach then will provide some motivating examples which will show the functional aspects of the approach proposed by us.

\section{CASE STUDY HOUSE BIDDING SYSTEM:}

A trivial House bidding does not use any kind of an online booking system. Booking or bidding is usually done by phone or by meeting in person, or sometimes by email also. This is not very effective or efficient as it wastes lot of time. Both Buyer and seller have to take time from their everyday life to talk to each other for fixing rates. My solution to this problem is an easy to use online web-based House bidding and management system. This system saves time and is more convenient to all. In this application houses are made available to potential bidders who can place bids on them. A bid is the amount of money for which a bidder is willing buy the house. An administrator will review bids and can accept one per House, in which case the house becomes unavailable to other bidders. This system is an exploration for integrating different web applications (like ASP.Net, Java, Silverlight) using web services concept. Language for communication between different web services will be XML (EXTENSIBLE MARKUP LANGUAGE). If format of $\mathrm{xml}$ generated is different for different applications then inbuilt XSLT transformation engine is used for mapping values. This case study will use the technologies like SOA, ASP.NET, JAVA, XML, XSLT, MS SQL Server 2000, Silverlight and WEB SERVICES.

The main topics that we researched on are:

SOA(Service oriented architecture)

Web services (soap and rest).

$\square \quad$ XML( EXTENSIBLE MARKUP LANGUAGE)

\section{CASE STUDY DESCRIPTION:}

It was decided to develop an application for house bidders, which will have two different types of users

- House administrators

- $\quad$ Bidders

This system will need to provide the following functionalities:

\section{ASP.NET Application:}

House administrators:

- Enter new and manage existing Houses for sale.

- $\quad$ Register new and manage existing bidders.

- View bids

- Accept a bid for a house.

Bidder: Bidders will use SOAP C\# web service to talk to database or House administrators.

- $\quad$ List and search for houses

- Place a bid for a house

\section{MODULE DESCRIPTION}

Database: The database represents the data tier of the system. The database will store the information required for houses, bidders, bids etc. The database we used is Microsoft SQL Server.

Managing Houses: We will be implementing a .NET application that allows the administrator to add, edit and delete houses. This application will use the database described above.

Registering Bidders: We will be extending above application, such that it is possible for the administrator to register new bidders and edit/delete existing ones.

Searching and Bidding houses: Here we will develop a Java web application which allows a user to log in, then search for houses and place bids on house. There will be 3 search 
criteria: Postcode, address where the house is located and cost of house.

The application will search for the information given and return a list of matching houses. It should be possible to leave some search criteria empty, in which case they should be ignored. If all criteria are empty, then all houses will be returned.

You will be able to place a bid over the houses. A bid will be identified by the bidder who placed the bid, the house for which the bid was made and the amount.

$\square \quad$ Accepting Bids: Here administrator will accept bids that have been made. Only one bid can be accepted for each house and as soon as a bid is accepted, the house should not be available to other bidders i.e. it should not be returned in search results.

$\square \quad$ This application is extendable you can add more modules as per the need, later in future.

C\# Soap web service: This web service will read database and then will exposes three web

Method - Login, Search, InsertBid.

Login: Login will be exposed so that whenever a bidder wants to bid they can use their login credentials and then can bid. This will save our system from unwanted traffic of clients, only registered customers can bid.

Search: Using this functionality any bidder can search for the available house in our database with the requirements postcode, address and cost. These three search criteria are basic for searching any house you can add or delete these search fields depending upon the user requirements.

InsertBid: This functionality was provided for inserting the bid by bidders in our database. So the details of bidder and bid can be inserted in the database.

Java client application: This application will use the web methods exposed by soap web service. This application will be used by bidders to insert bids for the houses. They have to first login and then they can search for the house and insert bid on it. Some of the client side and server side validation will be added later in this part. So now any buyer can login and search for house and then can place bids over these houses. Once the bid is accepted it won't be shown again in search criteria.

RestWebservice: We will consume SOAP web service by REST Web service. This web service will again expose search methods provided by above SOAP web service i.e. it will not talk to database directly but it can interact using SOAP web service. This will be one type of implementation you can try any combinations according to the requirements.

SilverLight Application: At last this RESTWebservice is consumed by silverlight application. To make a silverlight application we will design its User interface using Microsoft blend and then save it, this will automatically be there when you open same application in Asp.Net. Later we can develop code for consuming your RESTWebservice. There are many more technologies which can consume REST web service and can be used in future as per the requirements. We have used SilverLight as programmers can code in ASP.NET their silverlight application and designer can design using Microsoft Blend.

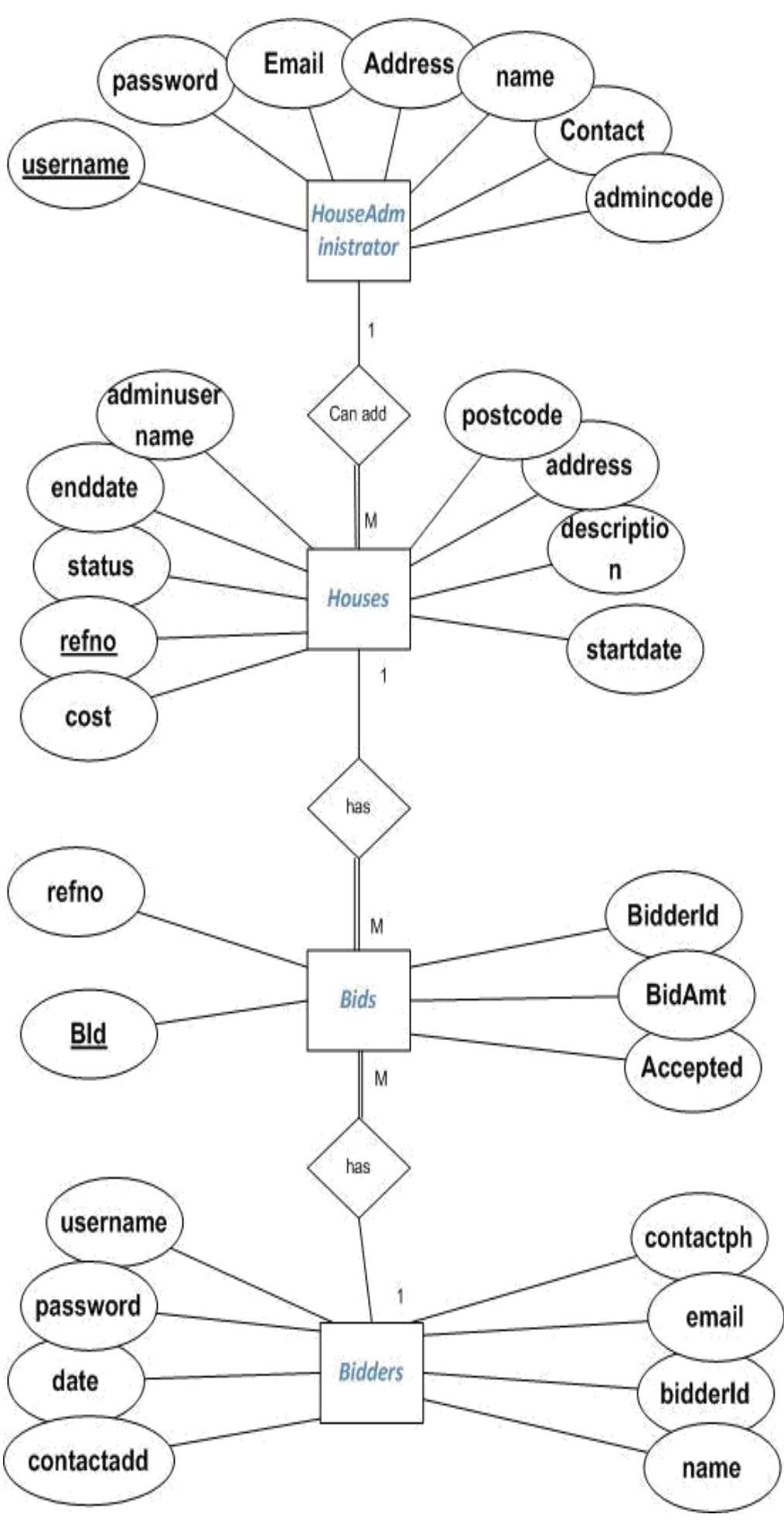

Fig 2. ERD( Entity relationship diagram) 


\section{IMPLEMENTATION}

The first and foremost step in implementation is research of similar topics available in the market. So for developing this case study we have to refer to the other developer's work, read different articles and have to learn from different tutorials. The next phase is to determine how all functionalities will be implemented in most effective and efficient way. For this, different functionalities that are either useful for customer or for administrator will be implemented, but still there can be many more things that can be added later (these are described in further work). This case study is just a prototype so that we can implement any sort of such distributed applications in most effective and efficient way as possible.

Finally it was decided that the application should be made in ASP.NET and using direct call to C\# SOAP web services using java code. SQL server is used rather than MS Access because it is much more scalable and has better security. We will use ASP.NET as it is very convenient for me to make application in this. And also there is no need to write any HTML code, in this everything is generated by IDE (Integrated Development Environment). It is very easy to change properties of anything using controls provided. It is also very simple to write code or manage code in this as both design and coding are separated as different files. So designers can do their work and developer has to concentrate on just theirs.

To start with the implementation of the case study, we first decided to make basic integrations like how asp.net communicates with web service or java application is not connect to the database directly, but only access it via the web service. Here are some screen shots of these integrations:

\section{Case study: House Bidding application}

\section{HouseBiddingWS:}

This is a SOAP web service which will expose 3 methods login, search and InsertBid. When you click Login you will get the screen

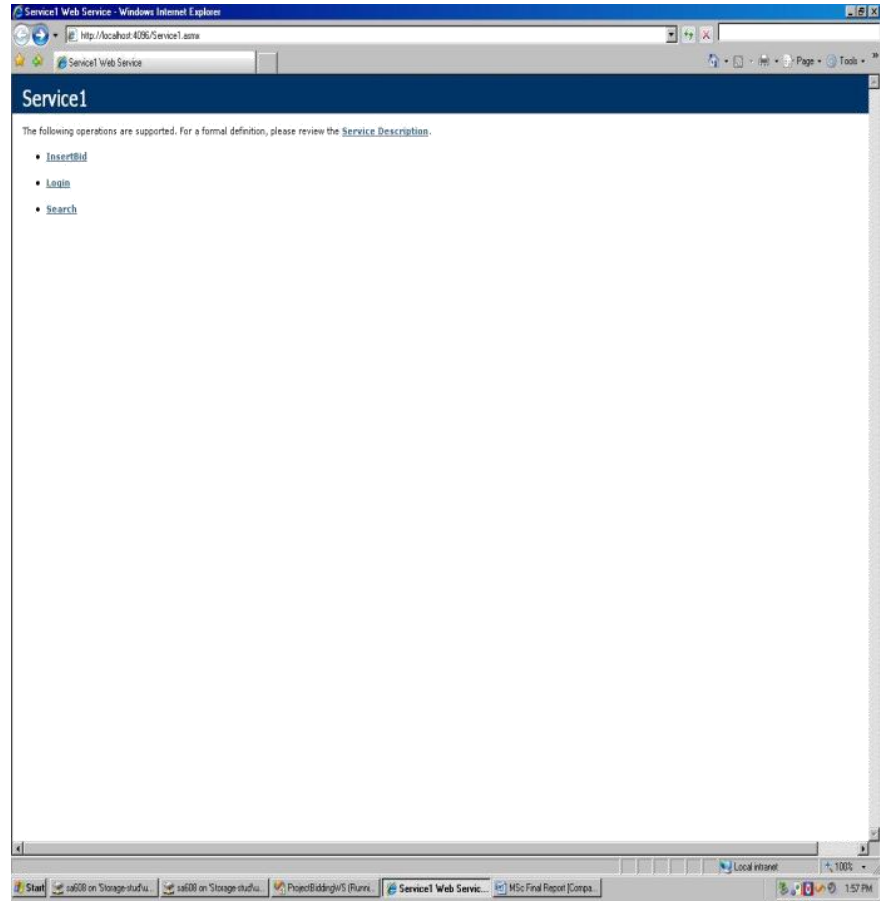

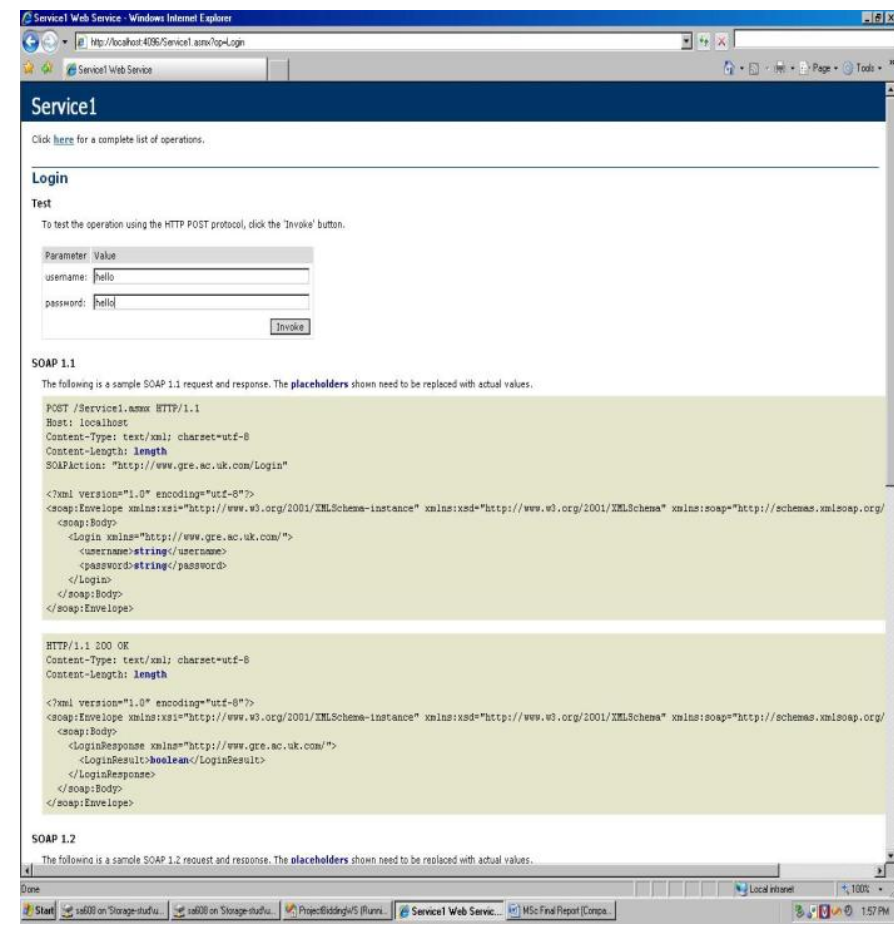

Click on Invoke to invoke this web method of Web service. This method will check from database for login and password and return true or false depending upon it.

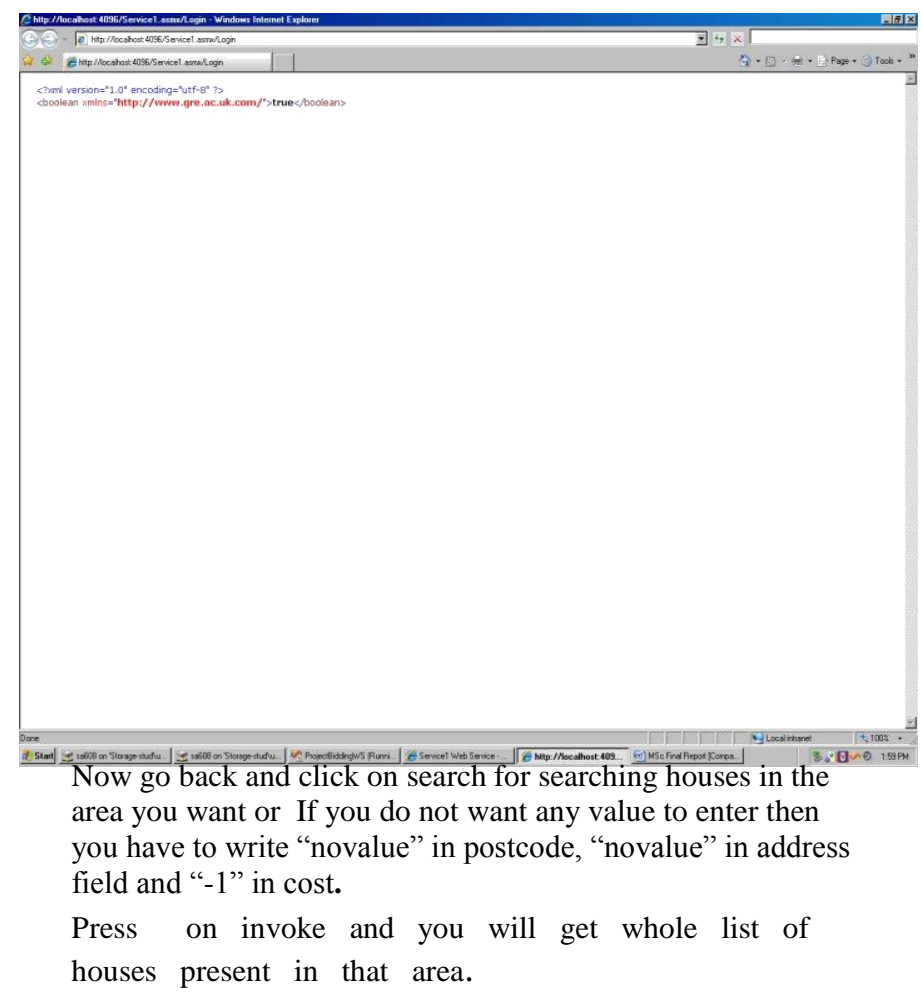




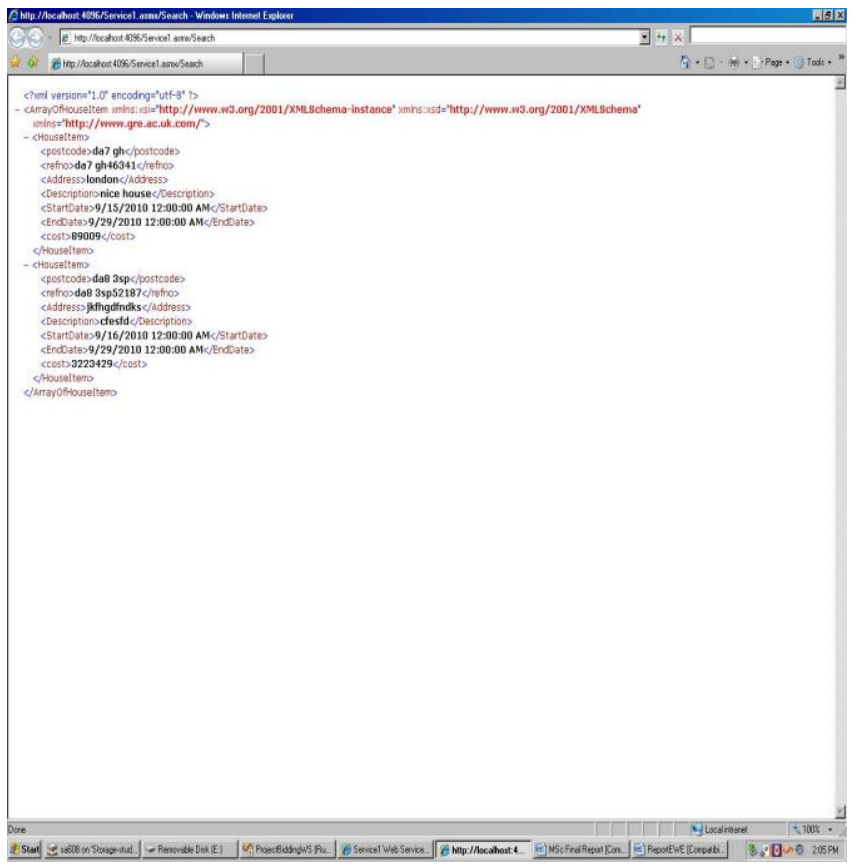

Next functionality is InsertBid for placing bids by bidders or you can say buyers.

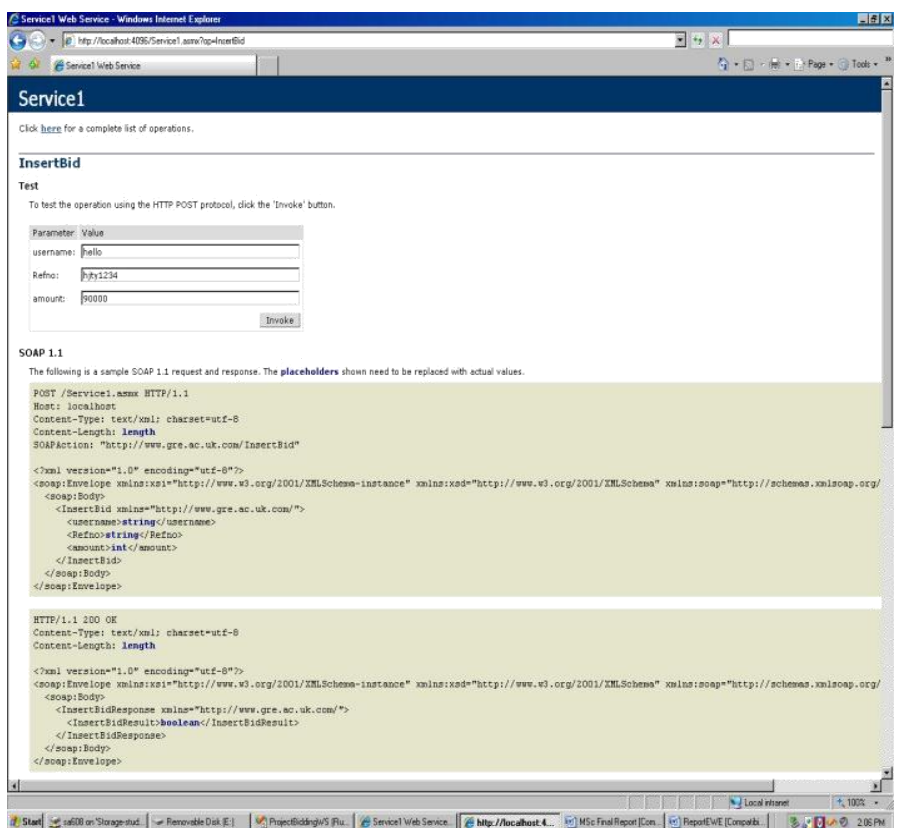

Give values and click on invoke you will get a boolean value as result.

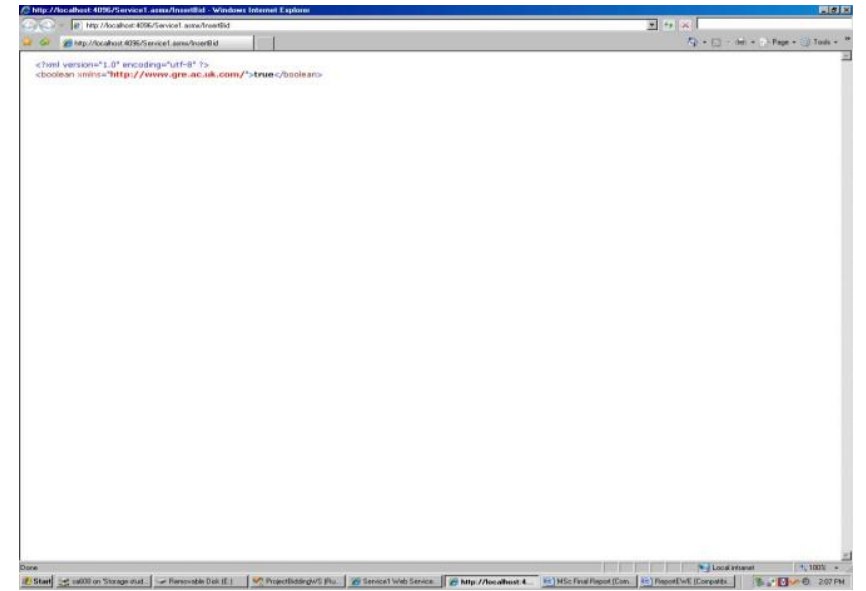

\section{RestWSBiddingSystem:}

This is a rest web service which will further use SOAP web service and expose its search method so that it can be used by other applications which support REST web services. Click on Rest WS Bidding System icon and enter value for postcode, address and cost.
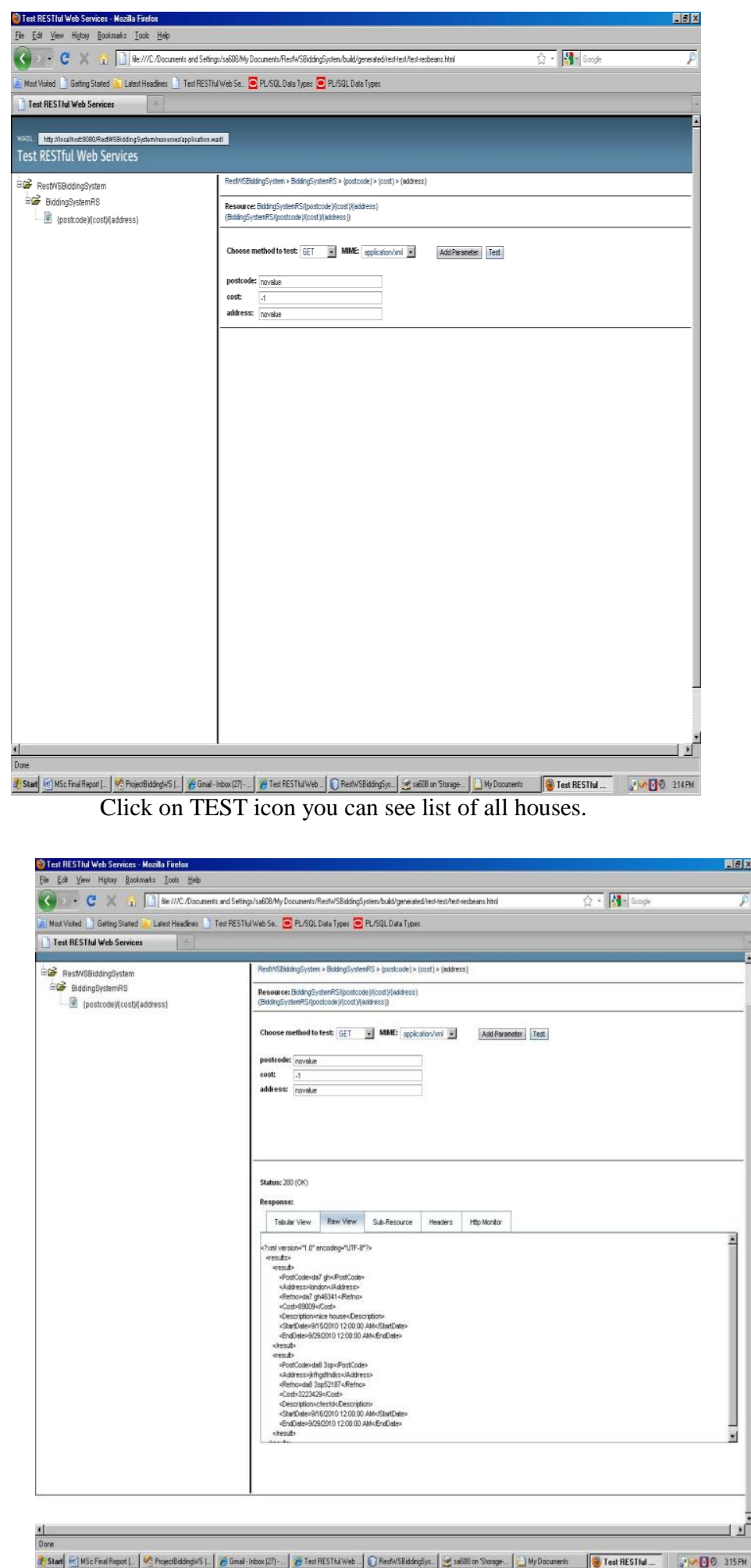

Here you can change the view for seeing your output also. 
Also make sure you are using Mozilla to run this REST web service as internet explorer does not support it properly.

Now this REST web service can be consumed by Silverlight application later in the case study.

\section{SUMMARY}

In this paper, we proposed an approach of integrating heterogeneous web applications. We first describe few terminologies and then we decided what all functionalities our case study will have and which technologies will be used and how. We have also described our database and all its entities using an ERD [Entity Relationship Diagram]. Later on we showed some experiments on web services and a working C\# SOAP web service using java code and REST web service is made .These web services will be used later to integrate final application. We will give all possible efforts to make application, flexible, smooth, clear and straight forwarded.

\section{FURTHER WORK}

There is tremendous scope for further progression of this paper. These types of applications can be used for making any small or big business applications just by making few more changes to it. Because of the potential benefits of web service, future research will contribute to many business areas. The application will be vulnerable to security attacks and future research will help to deal it. Another interesting issue is to extend our architecture which we will deal later on. However, further research is necessary to apply the solution effectively.

\section{REFERENCES}

[1] Thomas, E. (2005),'Service-Oriented Architecture: Concepts, Technology, and Design', www.soaprinciples.com

[2] Hugo, H. et. al.(2002) 'Web Services Glossary',http://www.w3.org/TR/ws-gloss

[3] Unknown,http://publib.boulder.ibm.com/infocenter/iadth elp/v6r0/index.jsp?topic=/com.ibm.

[4] etools.webservice.doc/concepts/csoap.html

[5] Sabbouh, M. et. al.(2001),'Workshop on Web services',http://www.w3.org/2001/03/WSWSpopa/paper08

[6] Author unknown (2006), 'Proceedings of the 28th international conference on Software engineering', ICSE'06,20,Pp. 20-28 .

[7] Plew R. R. et al(2004), Teach Yourself SQL in 21 Days,SAMS.

\section{Internet references: <internet>}

www.soaprinciples.com

http://download.microsoft.com/download/SOA.ppt

http://quickstarts.asp.net/QuickstartV20/aspnet/

www.codeproject.com/vb/net/

www.asp.net.com

http://www.oracle.com/us/technologies/029118.pdf

http://www.buzzle.com/editorials/4-10-2005-68349.asp 